\title{
1 Title: Genome sequencing and assembly of Tinospora cordifolia (Giloy) plant
}

2 Authors: Shruti Mahajan, Abhisek Chakraborty, Titas Sil, Vineet K Sharma*

3 Affiliation: MetaBioSys Group, Dept. of Biological Sciences, Indian Institute of Science Education

4 and Research Bhopal, Bhopal, Madhya Pradesh, 462066, India

$5 \quad *$ Corresponding Author email:

$6 \quad$ Vineet K Sharma - vineetks@iiiserb.ac.in

8 E-mail addresses of authors: Shruti Mahajan - shruti17@iiserb.ac.in, Abhisek Chakraborty 9 abhisek18@iiserb.ac.in, Titas Sil - titas16@iiserb.ac.in, Vineet K Sharma - vineetks@iiserb.ac.in 
During the ongoing COVID-19 pandemic Tinospora cordifolia also known as Giloy gained immense popularity and use due to its immunity-boosting function and anti-viral properties. T. cordifolia is among the most important medicinal plants that has numerous therapeutic applications in health due to the production of a diverse array of secondary metabolites. Therefore, to gain genomic insights into the medicinal properties of $T$. cordifolia, the first genome sequencing was carried out using $10 \mathrm{x}$ Genomics linked read technology and the draft genome assembly comprised of $1.01 \mathrm{Gbp}$. This is also the first genome sequenced from the plant family Menispermaceae. We also performed the first genome size estimation for T. cordifolia and was found to be $1.13 \mathrm{Gbp}$. The deep sequencing of transcriptome from the leaf tissue was also performed followed by transcriptomic analysis to gain insights into the gene expression and functions. The genome and transcriptome assemblies were used to construct the gene set in $T$. cordifolia that resulted in 19,474 coding gene sequences. Further, the phylogenetic position of $T$. cordifolia was also determined through the construction of a genome-wide phylogenetic tree using 35 other dicot species and one monocot species as an outgroup species.

\section{INTRODUCTION}

Tinospora cordifolia is a climbing shrub belonging to the Menispermaceae family that includes more than 400 plant species of high therapeutic properties $[1,2]$. It perhaps originated in Africa in the Oligocene epoch (28.57 million years ago) and was spread to Asia in the early Miocene epoch (21.54 million years ago) [3]. T. cordifolia is found in tropical and sub-tropical regions including India, China, Sri Lanka, Bangladesh, Myanmar, Thailand, Malaysia, etc. and also known as 'Giloy', 'Amrita', 'Guduchi', and 'heart leaved moonseed' [2]. It is a perennial deciduous dioecious plant with morphological characteristics of twining branches, succulent stem with papery bark, alternatively arranged heart-shaped leaves, aerial roots and greenish yellow tiny flowers in the form of racemes inflorescence [2, 4]. Being a climber, T. cordifolia needs a supportive plant like Jatropha curcas (Jatropha), Azadirachta indica (Neem), Moringa oleifera (Moringa), etc. for its growth [4]. These cooccurring plants also play an important role in enhancing the production of various secondary metabolites of T. cordifolia $[4,5]$. Previous reports also indicated the presence of endophytic fungi in the leaves and the stem of this plant but their ecological significance has yet to be studied [6,7]. This plant produces the secondary metabolites in response to the stress conditions and their concentration also varies based on seasons and its dioecy status [8]. High genetic diversity has been reported in $T$. cordifolia due to the dioecious nature [9-11]. 
42 The chemical constituents of this plant have been broadly categorized as alkaloids (tinosporine, 43 magnoflorine, berberine, etc.), terpenoids (tinosporide, furanolactone diterpene, cordifolioside, etc.), 44 phenolics (lignans, flavonoids, phenylpropanoids etc.), polysaccharides (glucose, xylose, rhamnose, etc.), steroids (giloinsterol, ß-sitosterol, etc.), essential oils and aliphatic compounds along with a few other compounds such as giloin, tinosporidine, sinapic acid, tinosporone, tinosporic acid, etc. that are obtained from various parts of the plant $[12,13]$. A terpene tinosporaside and an alkaloid berberine were found to be the most dominant compounds in $T$. cordifolia and suggested to use them as its chemical biomarkers $[14,15]$. The bitter taste of $T$. cordifolia is due to the presence of tinosporic acid, tinosporol, giloin, giloinin, tinosporide, cordifolide, tinosporin and a few other compounds [12]. A study reported that among the two species of Tinospora (i.e. T. cordifolia and T. sinensis), T. cordifolia produces three times higher concentration of berberine than $T$. sinensis, and thus the former is preferred in therapeutics [16].

The bioactive compounds found in T. cordifolia have known biological properties such as anti-pyretic, anti-diabetic, anti-inflammatory, anti-microbial, anti-allergic, anti-oxidant, anti-diabetic, anti-toxic, anti-arthritis, anti-osteoporotic, anti-HIV, anti-cancer, hepatoprotective, anti-malarial, and also in immunomodulation etc. $[17,18]$. These properties make this species useful in the traditional treatment of several ailments including fevers, cough, diabetes, general debility, ear pains, jaundice, asthma, heart diseases, burning sensation, bone fracture, urinary problems, chronic diarrhoea, dysentery, leucorrhoea, skin diseases, cancer, helminthiasis, leprosy and rheumatoid arthritis. Further pre-clinical and clinical studies have been carried out to indicate its potential to treat leucopenia induced by breast cancer chemotherapy, hepatic disorders, post-menopausal syndrome, obstructive jaundice, etc. [19, 20]. These diverse and important therapeutic applications make it a species of interest for a broad scientific community. Interestingly, this Giloy plant has gained tremendous therapeutic interest and significance during the recent and ongoing COVID-19 pandemic [21].

However, despite the widely known and important medicinal properties of this plant its genome assembly is yet unavailable. A preliminary study reported the transcriptome (482 Mbp data) of this species from leaf and stem tissues using 454 GS-FLX pyrosequencing [22]. A recent karyological study reported $2 \mathrm{n}=26$ as the chromosome number in $T$. cordifolia, which was also supported by the earlier studies [23-25]. Thus, to uncover the genomic basis of its medicinal properties and for further exploration of its therapeutic potential, we carried out the first genome sequencing and assembly of $T$. cordifolia using 10x Genomics linked reads. This is the first draft genome assembly of T. cordifolia which is also the first genome sequenced so far from the medicinally important genus Tinospora and its family [26]. We also carried out a comprehensive deep sequencing and assembly of the leaf 
75 transcriptome using Illumina reads. The genome-wide phylogenetic analysis was also carried out for

76 T. cordifolia with other dicot species and a monocot species as an outgroup to determine its

77 phylogenetic position.

\section{METHODS}

Sample collection, species identification, nucleic acids extraction and sequencing

81 The plant was brought from a nursery in Bhopal, Madhya Pradesh, India $\left(23.2599^{\circ} \mathrm{N}, 77.4126^{\circ} \mathrm{E}\right)$.

82 DNA and RNA extraction were carried out using a cleaned leaf, which was homogenized in liquid 83 nitrogen. The TRIzol reagent was used for RNA extraction [27]. For genomic DNA extraction, the 84 powdered leaf was washed with $70 \%$ ethanol and distilled water in order to eliminate any such 85 compounds that may hinder the extraction process and employed CTAB based Carlson lysis buffer for 86 the isolation [28]. Two genes: one nuclear gene and one chloroplast gene (Internal Transcribed Spacer 87 and Maturase K, respectively) were used for the species identification. These genes were amplified and sequenced at in-house sanger sequencing facility. The TruSeq Stranded Total RNA library preparation kit with Ribo-Zero Plant workflow (Illumina, Inc., USA) was deployed for preparing the transcriptomic library. The genomic library for linked reads was prepared using Gel Bead kit and Chromium Genome library kit on a Chromium Controller instrument (10x Genomics, CA, USA). The quality of both the libraries (transcriptomic and genomic) was checked on Tapestation 4150 (Agilent, Santa Clara, CA) and sequenced on an Illumina platform, NovaSeq 6000 (Illumina, Inc., USA) for producing paired end reads. The comprehensive DNA and RNA extraction procedure is mentioned in Supplementary Text S1.

\section{Genome assembly}

An array of Python scripts (https://github.com/ucdavis-bioinformatics/proc10xG) were used to remove the barcode sequences from the raw reads. SGA-preqc was employed for genome size estimation of $T$. assembly was generated by Supernova assembler v.2.1.1 (with maxreads=all options and other default settings) using 499.36 million raw reads [30]. The 'pseudohap2' style in Supernova mkoutput was implemented to assemble haplotype-phased genome. 
104 The barcodes of linked reads were processed using Longranger basic v2.2.2 105 (https://support.10xgenomics.com/genome-exome/software/pipelines/latest/installation) and these

106

107

108

109

110

111

112

113

114

115

116

117

118

119

120

121

122

123

124

125

126

127

128

129

130

131

132

133

134

135 processed reads were used by Tigmint v1.2.1 to rectify the mis-assemblies present in Supernova assembled genome [31]. AGOUTI v0.3.3 with quality-filtered transcriptome reads was used to accomplish the initial scaffolding [32]. In order to construct a more contiguous assembly ARCS v1.1.1 with its default parameters was used to provide additional scaffolding and enhance the contiguity of the genome assembly [33]. Using a bloom filter-based method and k-mer value ranging from 30 to 120 with 10 bp interval, Sealer v2.1.5 used the linked reads (barcode processed) for gap-closing in the assembly [34]. Performing scaffolding multiple times could give rise to local mis-assemblies, small indels or distinct base errors which were overwhelmed using Pilon v1.23 that utilized the linked reads (barcode-processed) to increase the assembly quality [35]. The completeness of genome assembly was evaluated with BUSCO v4.1.4 which used embryophyte_odb10 database for the assessment [36]. The additional information about the post-processing of genome assembly is provided in Supplementary

\section{Text S2.}

\section{Transcriptome assembly}

The de novo transcriptome assembly was carried out using RNA-Seq data generated in this study. Trimmomatic v.0.38 was used for processing of raw data reads i.e., adapter removal and qualityfiltration [Supplementary Text S2] [37]. The de novo transcriptome assembly was constructed using Trinity v2.9.1 with strand-specific option and other default parameters using the processed paired-end reads [38]. A Perl script offered in Trinity software package was utilized to evaluate the assembly statistics.

\section{Genome annotation}

The genome annotation was achieved on the polished assembly (length $\geq 1,000 \mathrm{bp}$ ). This genome was used by RepeatModeler v2.0.1 to construct a de novo repeat library [39]. The clustering of obtained repeat sequences was performed using CD-HIT-EST v4.8.1 with sequence identity as $90 \%$ and 8 bp seed size [40]. Using the repeat library, RepeatMasker v4.1.0 (RepeatMasker Open-4.0, http://www.repeatmasker.org) was used to soft-mask the genome that was used for the construction of gene set. MAKER pipeline that employs ab initio-based gene prediction programs as well as evidencebased approaches for prediction of final gene model was used for genome annotation [41]. As an empirical evidence in MAKER pipeline the de novo transcriptome assembly of $T$. cordifolia and protein sequences of its phylogenetically closer species Beta vulgaris (belonging to plant order Caryophyllales) were used. The ab initio gene prediction, evidence-based alignments and polishing of 
136 alignments were achieved using AUGUSTUS v3.2.3, BLAST and Exonerate v2.2.0, respectively with 137 the MAKER pipeline [42, 43]. The completeness of the coding gene set was also assessed using 138 BUSCO v4.1.4 embryophyte_odb10 database. The tandem repeats detection, de novo tRNAs 139 prediction, de novo rRNAs prediction and miRNAs identification (homology based) were performed 140 using Tandem Repeat Finder (TRF) v4.09, tRNAscan-SE v2.0.7, Barrnap v0.9 141 (https://github.com/tseemann/barrnap) and miRBase database, respectively [44-46]. The detailed 142 information about genome annotation is provided in Supplementary Text S3.

\section{Orthogroups identification}

144 Among all the eudicot species accessible on Ensembl Plants release 48, a total of 35 species were selected by choosing one species from each offered genus. The 35 eudicot species along with an outgroup species, Zea mays were used for the identification of orthologs [47]. The 36 selected species along with the outgroup species were - Actinidia chinensis, Arabidopsis thaliana, Arabis alpina, Beta vulgaris, Brassica napus, Camelina sativa, Cannabis sativa female, Capsicum annuum, Citrullus lanatus, Citrus clementina, Coffea canephora, Corchorus capsularis, Cucumis melo, Cynara cardunculus, Daucus carota, Glycine max, Gossypium raimondii, Helianthus annuus, Ipomoea triloba, Lupinus angustifolius, Malus domestica Golden, Manihot esculenta, Medicago truncatula, Nicotiana attenuata, Olea europaea var. sylvestris, Phaseolus vulgaris, Pistacia vera, Populus trichocarpa, Prunus avium, Rosa chinensis, Solanum tuberosum, Theobroma cacao Matina 1-6, Trifolium pratense, Vigna angularis, Vitis vinifera, and Zea mays. The orthogroups were formed using the proteome files of 35 selected eudicot species along with Zea mays and MAKER retrieved protein sequences of T. cordifolia. Among all the protein sequences, the longest isoforms were retrieved for all the species and provided to OrthoFinder v2.4.1 for orthogroups construction [48].

\section{Orthologous gene set construction}

The orthogroups comprising genes from all 37 species were retrieved from all the identified orthogroups. KinFin v1.0 was used to increase the genes in one-to-one orthogroups that identified and extracted fuzzy one-to-one orthogroups among these retrieved orthogroups [49]. In cases where multiple genes were present for a single species in any orthogroup, the longest gene among them was selected as representative.

\section{Phylogenetic tree construction}

MAFFT v7.480 was used to discretely align all the identified fuzzy one-to-one orthogroups for construction of the phylogenetic tree [50]. The multiple sequence alignments were trimmed to 
167 eradicate empty sites, and the alignments were concatenated using BeforePhylo v0.9.0 168 (https://github.com/qiyunzhu/BeforePhylo). The concatenated alignments were used by RAxML $169 \mathrm{v} 8.2 .12$, based on rapid hill climbing algorithm, to create the maximum likelihood-based phylogenetic tree (100 bootstrap values and amino acid substitution model 'PROTGAMMAAUTO’) [51].

171

172

173

174

175

176

177

178

179

180

181

182

183

184

185

186

187

188

189

190

191

192

193

194

195

196

\section{RESULTS}

\section{Sampling and Sequencing of $T$. cordifolia genome and transcriptome}

The T. cordifolia plant was brought from a plant nursery in Bhopal, Madhya Pradesh, India and the DNA and RNA extracted from leaf was used for sequencing. Two marker genes: ITS and MatK were amplified and sequenced at in-house sanger sequencing facility for the species identification (Supplementary Text S1). The sequenced reads of these marker genes were aligned using BLASTN that showed the highest identity (98.32\% for ITS and $99.41 \%$ for MatK) with sequences of T. cordifolia available at NCBI database and confirmed the plant species as T. cordifolia. We generated $79.4 \mathrm{Gbp}$ of genomic data and $34.7 \mathrm{Gbp}$ of transcriptomic data for the genomic assembly and analysis of $T$. cordifolia (Table 1 and Supplementary Tables S1-S2). The barcode sequences were removed from the raw reads and high-quality reads were selected for further analysis. The de novo assembly generated by Supernova assembler v.2.1.1 using 499.36 million raw reads [30], and the 'pseudohap2' style in Supernova mkoutput was implemented to assemble haplotype-phased genome. Since the genome size was not known for this plant, we performed the first genome size estimation for $T$. cordifolia using SGA-preqc processed linked reads, and the genome size was estimated to be 1.13 Gbp. Considering this genome size, the sequenced genomic data amounts to $70.2 \mathrm{x}$ genome coverage. After scaffolding, mis-assemblies rectification, gap-filling and polishing T. cordifolia genome assembly resulted in $1.01 \mathrm{Gbp}$ assembly size $(\geq 2,000 \mathrm{bp})$ as the final draft. The $\% \mathrm{GC}$ of the assembled genome was $35.12 \%$, and a total of 56,342 scaffolds were obtained having the N50 of $50.2 \mathrm{Kbp}$ (Table 1 and Supplementary Table S3). The BUSCO completeness was $78.9 \%$ in the final polished $T$. cordifolia genome assembly (Supplementary Table S4). The de novo transcriptome assembly was constructed using Trinity v2.9.1 with strand-specific option and other default parameters using the processed paired end reads. The Trinity software assembled 2,764,154 bp of de novo transcriptome assembly, and a total of 8,208 transcripts were predicted in the transcriptome assembly.

\section{Annotation of genome and gene set formation}


197 The final polished genome assembly was analyzed by RepeatModeler v2.0.1 to construct a de novo 198 repeat library consisting of 1,918 repeat families that were further clustered into 1,584 representative repeat family sequences. The repetitive sequences in $T$. cordifolia genome were predicted using RepeatMasker v4.1.0 and obtained $75.15 \%$ of repetitive sequences in this genome. Among these repetitive sequences, $73.75 \%$ were characterized as interspersed repeats comprising $32.12 \%$ retroelements (29.15\% of LTR repeats), $2.48 \%$ DNA transposons and $39.14 \%$ unclassified repeats. The LTR repeats consisted of 25.38\% Gypsy/DIRS1 and 3.22\% Ty1/Copia elements. About 3.87\% of the genome comprised of simple repeats found using TRF v4.09. The interspersed and simple repetitive sequences collectively comprised $\sim 78 \%$ of the total genome. 392 hairpin miRNAs, 1,344 rRNAs and 2,186 tRNAs (decoding standard amino acids) were also predicted among the non-coding RNAs.

The soft-masked genome (generated using RepeatMasker) was used for the construction of gene set using the MAKER pipeline that employs ab initio-based gene prediction programs and evidence-based approaches for prediction of final gene model [41]. The de novo transcriptome assembly of $T$. cordifolia and protein sequences of its phylogenetically closer species Beta vulgaris (belonging to plant order Caryophyllales) were used as an empirical evidence in MAKER pipeline. The ab initio gene prediction, evidence-based alignments, and alignments polishing were achieved using AUGUSTUS v3.2.3, BLAST and Exonerate v2.2.0, respectively in the MAKER pipeline that predicted 19,730 coding sequences in the genome assembly [42,43]. These coding genes were filtered based on length $\geq 150$ bp and resulted in 19,474 coding gene sequences. The completeness of the coding gene set was assessed using BUSCO v4.1.4 embryophyte_odb10 database on the final gene set that showed $70 \%$ of the complete and fragmented BUSCOs to be present in the coding gene set.

\section{Phylogenetic analysis of $T$. cordifolia}

A total of 35 species were selected from the eudicot species accessible on Ensembl Plants release 48 by choosing one species from each offered genus, and included Actinidia chinensis, Arabidopsis thaliana, Arabis alpina, Beta vulgaris, Brassica napus, Camelina sativa, Cannabis sativa female,

223 Capsicum annuum, Citrullus lanatus, Citrus clementina, Coffea canephora, Corchorus capsularis, 224 Cucumis melo, Cynara cardunculus, Daucus carota, Glycine max, Gossypium raimondii, Helianthus 225 annuus, Ipomoea triloba, Lupinus angustifolius, Malus domestica Golden, Manihot esculenta, 226 Medicago truncatula, Nicotiana attenuata, Olea europaea var. sylvestris, Phaseolus vulgaris, Pistacia vera, Populus trichocarpa, Prunus avium, Rosa chinensis, Solanum tuberosum, Theobroma cacao 
229 with one outgroup species (Zea mays), and including T. cordifolia were used to detect 162,809

230 orthogroups using the protein sequences retrieved from 37 species.

231 The phylogenetic tree (maximum likelihood-based) was constructed using 454 fuzzy one-to-one 232 orthogroups predicted by KinFin v1.0 across the 37 species. The missing or unknown values were 233 predicted by aligning, concatenating and filtering all the fuzzy one-to-one orthogroups. The alignment 234 data was filtered resulting in 422,034 alignment positions. The phylogenetic tree was constructed using 235 this alignment data (filtered) of T. cordifolia along with the 35 dicot species available on Ensembl 236 Plants release 48 and an outgroup species, Zea mays (Figure 2). The resultant phylogenetic position 237 of T. cordifolia was observed as a separate clade from all the other eudicots and monocot outgroup 238 plausibly because the clade to which $T$. cordifolia belongs is considered as basal eudicots that diverged very early from the other eudicots $[52,53]$.

\section{DISCUSSION}

242 T. cordifolia is known to produce several phytochemicals as secondary metabolites such as alkaloids, 243 terpenoids, tannins, phenolic compounds, steroids, flavonoids, phytosterols, volatile oils, etc. that are 244 responsible for its diverse medicinal properties like anti-oxidant, immunomodulatory, anti-microbial, 245 anti-viral, anticancer, anti-pyretic, anti-inflammatory, hepatoprotective, neuroprotective, anti246 osteoporotic, anti-toxic, anti-diabetic, anti-arthritis, anti-ulcer, etc. [18, 54] (Figure 1). Tinosporaside, 247 tembetarine and phenolic compounds were suggested to responsible for its anti-inflammatory, anti248 diabetic and anti-oxidant activities, respectively [55-57]. Likewise, a few other compounds from 249 Tinospora cordifolia were found accountable for its various activities [58-62]. Being a medicinally 250 important herb with therapeutic applications in multiple health conditions, the genome and 251 transcriptome sequencing of Tinospora cordifolia was much needed to gain insights into its genetic 252 information, transcriptome, and functional analysis of pathways associated with the secondary 253 metabolites production responsible for its medicinal properties. This study reported the first draft 254 genome assembly of $T$. cordifolia which is also the first genome from the genus Tinospora and its 255 family. This study also deliberates the genome assembly, transcriptome assembly, gene set and 256 phylogeny of $T$. cordifolia that will help in understanding the medicinal properties of this multipurpose 257 plant. The linked read sequencing has recently emerged as a promising technology to significantly 258 increase the contiguity in the genome assembly by increasing scaffold N50 and decreasing the number 259 of scaffolds compared to short read technology, and was successfully used for the sequencing of $T$. 260 cordifolia [63]. 
261 T. cordifolia belongs to order Ranunculales and our phylogenetic tree finds its position as a distinct

262 branch separate from all the other eudicots. This could be due to the early-divergence of order

263 Ranunculales from all the other core eudicots. Order Ranunculales is considered as an early-diverging

264 eudicot order and is among a few other eudicot orders (collectively known as basal eudicots) that are

265 found to be sister lineage to the core eudicots [52, 64, 65], which was also observed in the case of $T$.

266 cordifolia that showed early divergence from all other dicot species (Figure 2). Thus, the early

267 divergence could be the reason for its distinct position relative to the other eudicots and monocot

268 species. The correctness of this phylogeny is supported by the fact that the species belonging to the

269 same clade shared common nodes, e.g., Arabidopsis thaliana, Camelina sativa, Brassica napus, and

270 Arabis alpina shared the same clade because they belong to the same order Brassicales, and similarly

271 Gossypium raimondii, Theobroma cacao Matina 1-6 and Corchorus capsularis belong to order

272 Malvales and shared the same node in the phylogeny. Likewise, the members of order Fabales and

273 order Solanales also shared their respective nodes.

274

\section{CONCLUSION}

276 Thus, as apparent from the above literature-based evidences on the medicinal and pharmaceutical

277 properties of this plant, the availability of $T$. cordifolia genome will help in bridging the missing link

278 between its genomic and medicinal properties and provide leads for exploring the genomic basis of

279 these properties. It will also aid in various comparative genomic studies and will act as a reference for

280 the future species sequenced from its genus and family. It will also help in the genome-wide

281 phylogenetic assessments as well as evolutionary analyses on this species. The knowledge of

282 mechanisms and pathways involved in production of its numerous medicinally important secondary

283 metabolites will help in better exploitation of these pathways and resultant metabolites for medicinal

284 purposes and therapeutic applications.

\section{LIST OF ABBREVIATIONS}

287

COVID

Corona Virus Disease

$288 \mathrm{Gbp}$

Giga base pair

289 RNA

Ribonucleic Acid

$290 \mathrm{Mbp}$

Mega base pair 


$\begin{array}{lll}293 & \text { tRNA } & \text { Transfer RNA } \\ 294 & \text { rRNA } & \text { Ribosomal RNA } \\ 295 & \text { miRNA } & \text { micro-RNA } \\ 296 & \text { TRF } & \text { Tandem repeat finder } \\ 297 & \text { ITS } & \text { Internal transcribed spacer } \\ 298 & \text { MatK } & \text { Maturase K } \\ 299 & \text { BLAST } & \text { Basic Local Alignment Search Tool } \\ 300 & \text { BLASTN } & \text { Nucleotide BLAST } \\ 301 & \text { NCBI } & \text { National Center for Biotechnology Information } \\ 302 & \text { bp } & \text { Base pair } \\ 303 & \text { kbp } & \text { Kilo base pair } \\ 304 & \text { LTR } & \text { Long terminal repeats } \\ 305 & \text { DIRS1 } & \text { Dictyostelium intermediate repeat sequence 1 }\end{array}$

\section{COMPETING INTERESTS}

308 The authors declare no competing financial and non-financial interest.

\section{AUTHORS' CONTRIBUTIONS}

310 VKS perceived and coordinated the project and collected the plant sample. SM extracted the nucleic 311 acids (DNA and RNA) from the collected sample, prepared the DNA and RNA samples for

312 sequencing, and carried out the species identification assay. AC and VKS conceived the computational 313 outline of the study. AC accomplished all the computational analysis presented in the study. SM, AC 314 and VKS interpreted the results. SM and AC constructed the figures. SM, AC, TS and VKS wrote the 315 manuscript. All the authors have read and approved the final version of the manuscript.

\section{ACKNOWLEDGEMENT}

317 SM and AC acknowledge Council of Scientific and Industrial Research (CSIR) for supporting research 318 fellowships. TS thanks the Department of Science and Technology and IISc Bangalore for providing 319 the KVPY scholarship. The authors also thank IISER Bhopal for providing the intramural research 320 funds. 


\section{DATA AVAILABILITY}

322 The raw data has been submitted on NCBI database with BioProject accession number

323 PRJNA749156, Biosample accession SAMN20355817, and SRA accessions SRR15221491 and

324 SRR15221490.

\section{FIGURES}

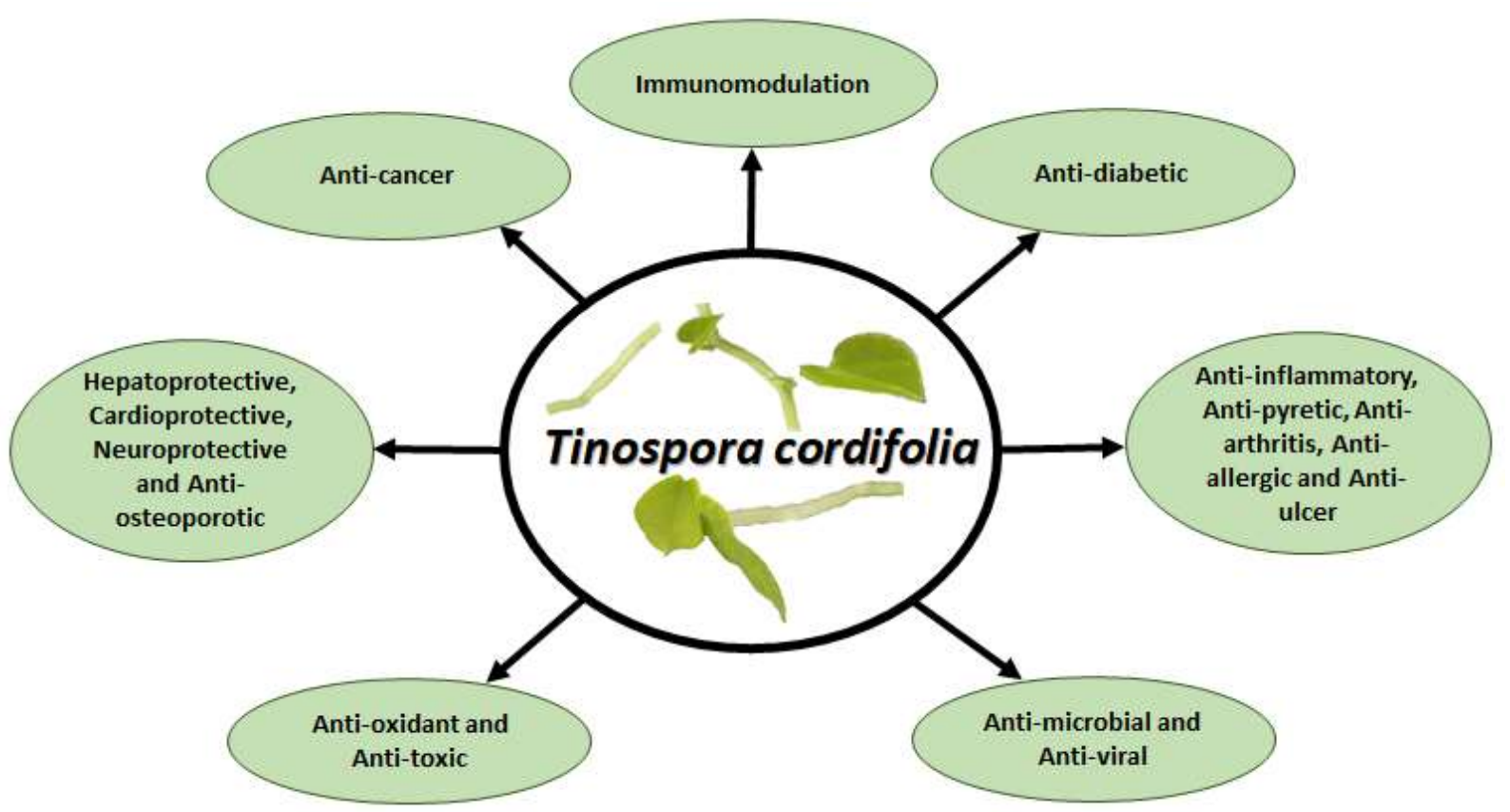

Figure 1. Medicinal properties of Tinospora cordifolia 


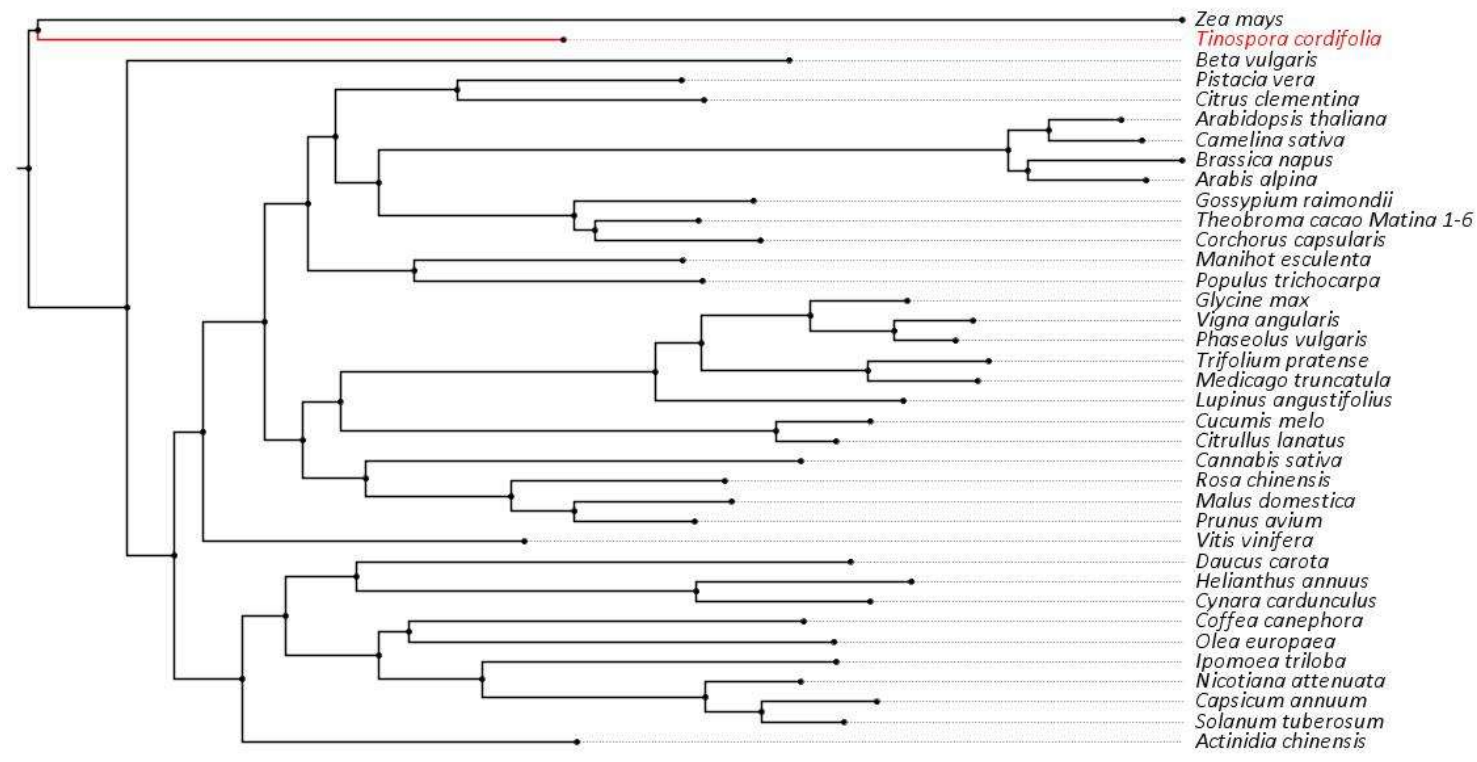

Figure 2. Phylogenetic tree describing the phylogenetic position of Tinospora cordifolia with other species

TABLE

333 Table 1. Summary of genome and transcriptome assemblies

\begin{tabular}{|c|c|}
\hline Parameter & Value \\
\hline Genomic data generated (Gbp) & 79.4 \\
\hline Estimated genome size (Gbp) & 1.13 \\
\hline Estimated genome coverage (x) & 70.2 \\
\hline Size of draft genome assembly (Gbp) & 1.01 \\
\hline N50 of genome assembly (Kbp) & 50.2 \\
\hline GC content (\%) & 35.12 \\
\hline Percentage of repetitive sequences (\%) & 75.15 \\
\hline Total coding sequences & 19,474 \\
\hline Transcriptome data generated (Gbp) & 34.7 \\
\hline Size of transcriptome assembly (bp) & $2,764,154$ \\
\hline Total transcripts identified & 8,208 \\
\hline
\end{tabular}

\section{REFERENCES}

1. Thulasi, M.M. and R.K. Vasu, The Menispermaceae family of plants and its action against infectious diseases: A review. Mapana Journal of Sciences, 2020. 19(2): p. 33-72.

$3413 . \quad$ Lian, L., et al., Re-delimitation of Tinospora (Menispermaceae): Implications for character evolution 
343 4. Mittal, J., M.M. Sharma, and A. Batra, Tinospora cordifolia: a multipurpose medicinal plant-A. Journal of Medicinal Plants, 2014. 2(2).

5. Sonavale, R., et al. EFFECT OF EDAPHIC FACTORS ON MAJOR SECONDARY METABOLITES OF TINOSPORA CORDIFOLIA AND NEEM GUDUCHI WITH RESPECT TO THEIR IMMUNOMODULATORY EFFECT. in International Conference on Contemporary Research in Chemical and Life Sceince. 2015.

6. Mishra, Y., et al., In vivo and in vitro histological localization of endophytic fungi in Tinospora cordifolia (Willd.) Miers ex Hook F. \& Thomas. Journal of Applied Research on Medicinal and Aromatic Plants, 2015. 2(1): p. 30-33.

7. Kapoor, N. and S. Saxena, Endophytic fungi of Tinospora cordifolia with anti-gout properties. 3 Biotech, 2018. 8(6): p. 1-6.

8. Choudhry, N., et al., Impact of seasons and dioecy on therapeutic phytoconstituents of Tinospora cordifolia, a Rasayana drug. BioMed research international, 2014. 2014.

9. Kalpesh, I. and J. Mohan, Assessment of genetic diversity in medicinal climber of Tinospora cordifolia (Willd.) Miers (Menispermaceae) from Gujarat, India. Asian Journal of Biotechnology, 2009. 1(3): p. 93-103.

10. Paliwal, R., et al., Development of genomic simple sequence repeats ( $g$-SSR) markers in Tinospora cordifolia and their application in diversity analyses. Plant Gene, 2016. 5: p. 118-125.

11. Lade, S., et al., Diversity in a widely distributed dioecious medicinal plant, Tinospora cordifolia (Willd.) Miers. ex. Hook F. and Thomas. Current Science, 2018. 114(7): p. 1520.

12. Singh, D. and P.K. Chaudhuri, Chemistry and pharmacology of Tinospora cordifolia. Natural product communications, 2017. 12(2): p. 1934578X1701200240.

13. Sharma, P., et al., The chemical constituents and diverse pharmacological importance of Tinospora cordifolia. Heliyon, 2019. 5(9): p. e02437.

14. Gahlaut, A., A. Gothwal, and R. Dabur, TLC based analysis of allelopathic effects on tinosporoside contents in Tinospora cordifolia. J. Chem. Pharm. Res, 2012. 4: p. 3082-3088.

15. Choudhary, N., M. Siddiqui, and S. Khatoon, Pharmacognostic evaluation of Tinospora cordifolia (Willd.) Miers and identification of biomarkers. 2014.

16. Srinivasan, G., et al., HPLC estimation of berberine in Tinospora cordifolia and Tinospora sinensis. Indian journal of pharmaceutical sciences, 2008. 70(1): p. 96.

17. Upadhyay, A.K., et al., Tinospora cordifolia (Willd.) Hook. f. and Thoms.(Guduchi)-validation of the Ayurvedic pharmacology through experimental and clinical studies. International journal of Ayurveda research, 2010. 1(2): p. 112.

18. Saha, S. and S. Ghosh, Tinospora cordifolia: One plant, many roles. Ancient science of life, 2012. 31(4): p. 151.

19. Sinha, K., et al., Tinospora cordifolia (Guduchi), a reservoir plant for therapeutic applications: $A$ Review. 2004.

20. Devi, P., Role of Tinospora cordifolia in metabolic health disorders: An updated review. Himalayan Journal of Health Sciences, 2021: p. 15-23.

21. Chopra, D., B. Bhandari, and S. Dwivedi, Beneficial role of Indian medicinal plants in COVID-19. MGM Journal of Medical Sciences, 2021. 8(2): p. 166.

22. Singh, R., et al., De novo transcriptome sequencing facilitates genomic resource generation in Tinospora cordifolia. Functional \& integrative genomics, 2016. 16(5): p. 581-591.

23. Abraham, A., Chromosome number in Tinospora. Current Science, 1942. 11(7): p. $282-282$.

24. Mathew, P. Studies on the Menispermaceae. in Proceedings of the Indian Academy of SciencesSection B. 1958. Springer.

25. Santra, I., T. Halder, and B. Ghosh, Research article Somatic and gametic chromosomal characterization with fluorescence banding of Giloy (Tinospora cordifolia): A berberine synthesizing important medicinal plant of India. Caryologia. International Journal of Cytology, Cytosystematics and Cytogenetics, 2021.

26. Haque, M.A., I. Jantan, and S.N.A. Bukhari, Tinospora species: An overview of their modulating effects on the immune system. Journal of ethnopharmacology, 2017. 207: p. 67-85. 
27. Johnson, M.T., et al., Evaluating methods for isolating total RNA and predicting the success of sequencing phylogenetically diverse plant transcriptomes. Plos one, 2012. 7(11): p. e50226.

28. Gaytán-Oyarzún, C.S.-H.a.J.C., Two mini-preparation protocols to DNA extraction from plants with high polysaccharide and secondary metabolites. African Journal of Biotechnology, 2006. 5 (20): p. 1864-1867.

29. Simpson, J.T. and R. Durbin, Efficient de novo assembly of large genomes using compressed data structures. Genome research, 2012. 22(3): p. 549-556.

30. Weisenfeld, N.I., et al., Direct determination of diploid genome sequences. Genome research, 2017. 27(5): p. 757-767.

31. Jackman, S.D., et al., Tigmint: correcting assembly errors using linked reads from large molecules. BMC bioinformatics, 2018. 19(1): p. 1-10.

32. Zhang, S.V., L. Zhuo, and M.W. Hahn, AGOUTI: improving genome assembly and annotation using transcriptome data. GigaScience, 2016. 5(1): p. s13742-016-0136-3.

33. Yeo, S., et al., ARCS: scaffolding genome drafts with linked reads. Bioinformatics, 2018. 34(5): p. 725731.

34. Paulino, D., et al., Sealer: a scalable gap-closing application for finishing draft genomes. BMC bioinformatics, 2015. 16(1): p. 1-8.

35. Walker, B.J., et al., Pilon: an integrated tool for comprehensive microbial variant detection and genome assembly improvement. PloS one, 2014. 9(11): p. e112963.

36. Simão, F.A., et al., BUSCO: assessing genome assembly and annotation completeness with singlecopy orthologs. Bioinformatics, 2015. 31(19): p. 3210-3212.

37. Bolger, A.M., M. Lohse, and B. Usadel, Trimmomatic: a flexible trimmer for Illumina sequence data. Bioinformatics, 2014. 30(15): p. 2114-2120.

38. Haas, B.J., et al., De novo transcript sequence reconstruction from RNA-seq using the Trinity platform for reference generation and analysis. Nature protocols, 2013. 8(8): p. 1494-1512.

39. Flynn, J.M., et al., RepeatModeler2 for automated genomic discovery of transposable element families. Proceedings of the National Academy of Sciences, 2020. 117(17): p. 9451-9457.

40. Fu, L., et al., CD-HIT: accelerated for clustering the next-generation sequencing data. Bioinformatics, 2012. 28(23): p. 3150-3152.

41. Campbell, M.S., et al., Genome annotation and curation using MAKER and MAKER-P. Current protocols in bioinformatics, 2014. 48(1): p. 4.11. 1-4.11. 39.

42. Altschul, S.F., et al., Basic local alignment search tool. Journal of molecular biology, 1990. 215(3): p. 403-410.

43. Stanke, M., et al., AUGUSTUS: ab initio prediction of alternative transcripts. Nucleic acids research, 2006. 34(suppl_2): p. W435-W439.

44. Benson, G., Tandem repeats finder: a program to analyze DNA sequences. Nucleic acids research, 1999. 27(2): p. 573-580.

45. Griffiths-Jones, S., et al., miRBase: tools for microRNA genomics. Nucleic acids research, 2007. 36(suppl_1): p. D154-D158.

46. Chan, P.P. and T.M. Lowe, tRNAscan-SE: searching for tRNA genes in genomic sequences, in Gene Prediction2019, Springer. p. 1-14.

47. Bolser, D., et al., Ensembl plants: integrating tools for visualizing, mining, and analyzing plant genomics data, in Plant bioinformatics2016, Springer. p. 115-140.

48. Emms, D.M. and S. Kelly, OrthoFinder: phylogenetic orthology inference for comparative genomics. Genome biology, 2019. 20(1): p. 1-14.

49. Laetsch, D.R. and M.L. Blaxter, KinFin: software for Taxon-Aware analysis of clustered protein sequences. G3: Genes, Genomes, Genetics, 2017. 7(10): p. 3349-3357.

50. Katoh, K. and D.M. Standley, MAFFT multiple sequence alignment software version 7: improvements in performance and usability. Molecular biology and evolution, 2013. 30(4): p. 772-780.

51. Stamatakis, A., RAXML version 8: a tool for phylogenetic analysis and post-analysis of large phylogenies. Bioinformatics, 2014. 30(9): p. 1312-1313. 
445 52. Worberg, A., et al., Phylogeny of basal eudicots: Insights from non-coding and rapidly evolving DNA. Organisms Diversity \& Evolution, 2007. 7(1): p. 55-77.

53. Wang, W., et al., Phylogeny and classification of Ranunculales: Evidence from four molecular loci and morphological data. Perspectives in Plant Ecology, Evolution and Systematics, 2009. 11(2): p. 81-110.

54. Begum, H.J. and V. Ramamurthy, Evaluation of secondary metabolites and antioxidant activity of ethanolic leaves extract of Tinospora Cordifolia.

450

55. Deepika, P.L., et al., Analgesic, Antiinflammatory Activity of Tinospora Cordifolia (Guduchi) and Valeriana Wallichi (Tagara) In Albino Rats. IOSR Journal of Pharmacy and Biological Sciences, 2016. Volume 11(Issue 2 Ver. IV): p. 18-22.

56. Khanal, P., et al., Network pharmacology-based assessment to elucidate the molecular mechanism of anti-diabetic action of Tinospora cordifolia. Clinical Phytoscience, 2019. 5(1).

57. Balkrishna, A., M.H. Kumar, and A.K. Gupta, COMPARATIVE ANALYSIS OF HPTLC, SECONDARY METABOLITES AND ANTIOXIDANT ACTIVITIES OF TINOSPORA CORDIFOLIA STEM POWDERS. International Journal of Pharmaceutical Sciences and Research, 2016. 7(10): p. 4263-4271.

58. Mishra, A., S. Kumar, and A.K. Pandey, Scientific validation of the medicinal efficacy of Tinospora cordifolia. The Scientific World Journal, 2013. 2013.

59. Borse, S., et al., Ayurveda botanicals in COVID-19 management: An in silico multi-target approach. Plos one, 2021. 16(6): p. e0248479.

60. Sharma, U., et al., Immunomodulatory active compounds from Tinospora cordifolia. Journal of ethnopharmacology, 2012. 141(3): p. 918-926.

61. Palmieri, A., et al., Berberine and Tinospora cordifolia exert a potential anticancer effect on colon cancer cells by acting on specific pathways. International journal of immunopathology and pharmacology, 2019. 33: p. 2058738419855567.

62. Bhutada, P., et al., Anticonvulsant activity of berberine, an isoquinoline alkaloid in mice. Epilepsy \& Behavior, 2010. 18(3): p. 207-210.

63. Mostovoy, Y., et al., A hybrid approach for de novo human genome sequence assembly and phasing. Nature methods, 2016. 13(7): p. 587-590.

64. Soltis, D., Angiosperm phylogeny inferred from $18 \mathrm{~S} r D N A, r b c L$, and atpB sequences. Botanical Journal of the Linnean Society, 2000. 133(4): p. 381-461.

65. Yang, L., et al., Phylogenomic Insights into Deep Phylogeny of Angiosperms Based on Broad Nuclear Gene Sampling. Plant Commun, 2020. 1(2): p. 100027. 